\title{
Pregnancy in Desmin-Related Cardiomyopathy
}

\author{
Arij Faksh, DO ${ }^{1} \quad$ Elisabeth Codsi, MD ${ }^{1} \quad$ Michel K. Barsoum, MD² Brian C. Brost, MD ${ }^{1}$ \\ ${ }^{1}$ Division of Maternal-Fetal Medicine, Department of Obstetrics and \\ Gynecology, Mayo Clinic, Rochester, Minnesota \\ 2 Department of Cardiology, Mayo Clinic Health System, Eau \\ Address for correspondence Arij Faksh, DO, Division of Maternal-Fetal \\ Medicine, Department of Obstetrics and Gynecology, Mayo Clinic, 200 \\ First St SW, Rochester, MN 55905 (e-mail: Faksh.arij@mayo.edu).
} Claire, Wisconsin

Am J Perinatol Rep 2015;5:e165-e167.

\begin{abstract}
Keywords

- pregnancy

- desminopathy

- restrictive cardiomyopathy

- DES gene

The course of desmin-related restrictive cardiomyopathy (DRCM) during pregnancy has not been described previously because of the rarity of the condition. Following an episode of heart failure antecedent to conception, a 28-year-old primigravida with DRCM presented to establish prenatal care during the first trimester. Prenatal management consisted of $\beta$-blocker and diuretic therapy, with serial echocardiography to monitor cardiac function. Spontaneous labor ensued at 39 weeks' gestation, and vacuum-assisted delivery was performed for fetal indication. Postpartum blood transfusion was required for symptomatic anemia because of uterine atony, and subsequent maternal and neonatal courses were uncomplicated. Cardiac evaluation postpartum demonstrated stable maternal status. Pregnancy in women with controlled DRCM is not contraindicated, however, it requires careful planning and monitoring during the antenatal, intrapartum, and postpartum periods. On the basis of this report, pregnancy does not appear to exert a permanent deleterious effect on cardiac function in women with DRCM.
\end{abstract}

Rarely encountered in the obstetric population, presenting symptoms of desmin-related myopathy include progressive myopathy, neuropathy, and cardiomyopathy between the second and fourth decades. ${ }^{1}$ Mutations (point substitutions, insertions, and deletions) in the DES gene results in altered myocyte intracellular structure, characterized by changes in nuclear shape, disturbances in mitochondrial morphology, abnormal accumulation of cytoskeletal proteins, and malalignment of myofibrils. ${ }^{1-3}$ Affected individuals initially experience lower extremity weakness and paresthesias, which may then extend insidiously into the trunk, neck, facial, and respiratory musculature. ${ }^{1,2}$ Intestinal and cardiac manifestations including arrhythmias or cardiomyopathy have been reported. ${ }^{4}$ The prevalence of DES mutations in dilated cardiomyopathy is rare ranging between 1 and $2 \%{ }^{5}$ Although most commonly inherited in an autosomal dominant manner or as a de novo/sporadic mutation, autosomal recessive patterns have rarely been described with variable phenotypic penetrance. $^{1}$

received

March 28, 2015

accepted after revision

May 14, 2015

published online

June 12, 2015
Formal diagnostic measures are based on clinical criteria of progressive muscle weakness, cardiomyopathy with/ without associated arrhythmia, or restrictive cardiac dysfunction, in conjunction with confirmatory molecular testing revealing characteristic DES gene mutation(s). ${ }^{1,2}$ To date, pregnancy has only been reported in a single patient with long QT syndrome and heart failure who was subsequently diagnosed postpartum with desmin-related cardiomyopathy (DRCM). ${ }^{6}$ This case report constitutes the first description of DRCM recognized before conception and managed prospectively.

\section{Case}

A 28-year-old primigravida was referred to us for consultation at $8^{5 / 7}$ weeks' gestation because of the DRCM diagnosed 2 years prior. Her medical history was significant for third-degree heart block requiring pacemaker placement 4 years previously; concurrent echocardiography

Copyright $\odot 2015$ by Thieme Medical Publishers, Inc., 333 Seventh Avenue, New York, NY 10001, USA. Tel: +1(212) 584-4662.
(요 (1) $\odot$
License terms
DOI http://dx.doi.org/ 10.1055/s-0035-1555130. ISSN $2157-7005$. 
demonstrated normal left ventricular ejection fraction (EF) of $60 \%$. Because of the symptomatic peripheral edema and intermittent arrhythmias, therapy with furosemide and carvedilol was instituted. Interestingly, the following year, her identical twin sister experienced a similar episode of third-degree heart block and cardiomyopathy and genetic testing was pursued revealing an Arg406Trp mutation in the DES gene, confirmatory for desmin myopathy. Repeat echocardiography in our patient 2 years after the initial episode showed left ventricular diastolic dysfunction with preserved EF. Comprehensive genetic evaluation in our patient revealed an identical mutation to her sister, and she was formally diagnosed with DRCM. Six months before the conception, a hospital admission was required for decompensated diastolic cardiac failure, which was successfully managed with the addition of an angiotensinconverting enzyme (ACE) inhibitor. In addition to the ACE inhibitor, before the pregnancy, she was managed on carvedilol and furosemide.

Following discovery of pregnancy, the ACE inhibitor was promptly discontinued, however, she remained on carvedilol and furosemide therapy. Although she met New York Heart Association class II functional criteria because of the persistent orthopnea with CARPREG score 2, ${ }^{7,8}$ echocardiography revealed a preserved left ventricular EF of $61 \%$ with a normal left ventricular chamber size. Given the impending physiologic increased cardiac demands of pregnancy, an uncertain prognosis was discussed. The patient elected to continue the pregnancy and declined the option of prenatal diagnosis for DRCM. Repeat maternal transthoracic echocardiogram at 19 weeks' gestation demonstrated stable cardiac function with normal left ventricular chamber size and $\mathrm{EF}$ of $57 \%$. Fetal echocardiogram was unremarkable. At 35 weeks' gestation, she was admitted for dyspnea with increasing peripheral edema and a repeat maternal echocardiogram showed preserved left ventricular EF of $58 \%$ with a normal left ventricular chamber size. Intravenous diuretic therapy was administered and she was maintained on carvedilol medication. She was readmitted the following week for similar symptoms without requiring further treatment.

Fetal growth was monitored sonographically on a monthly basis during the pregnancy and was consistently normal. At $39^{1 / 7}$ weeks' gestation, she presented in active labor, and a regional anesthetic was placed. Her intrapartum course was uneventful, and a low vacuum-assisted operative vaginal delivery was performed because of the category II fetal heart rate pattern late in the second stage of labor with delivery of a healthy female infant weighing $2,710 \mathrm{~g}$. Postpartum hemorrhage because of the uterine atony was encountered, and due to symptomatic hypotension and fall in hemoglobin from 11 to $7 \mathrm{~g} / \mathrm{dL}, 2$ units of blood were transfused. The remainder of her postpartum course was unremarkable, and both mother and infant were discharged home on the second day postdelivery. Neonatal echocardiogram and electrocardiogram were normal. Neonatal molecular genetic evaluation returned as positive for the mutation in the DES gene, Arg406Trp, the known disease causing mutation for myopathy and cardiomyopathy. Postpartum maternal cardiac evaluation revealed stable status without symptoms suggestive of cardiopulmonary exacerbation.

\section{Discussion}

At a cellular level, desmin functions as an intermediate filament cytoskeletal protein, playing a crucial role in cytoskeleton maintenance. Desmin links $Z$ bands and connects them to various intracellular structures including the sarcolemma, cytoplasmic organelles, and nuclear and plasma membranes. ${ }^{9}$ In affected individuals, the defective desmin transcript accumulates in a disorganized fashion, leading to a disrupted and abnormal formation of filaments. ${ }^{9}$ Myofibrillar myopathy or "desminopathy" is described as a familial or sporadic disorder from a mutation within the DES gene, leading to an abnormal accumulation of desmin protein within cardiac and skeletal muscles. ${ }^{1,2}$ Several other abnormalities include abnormal electromyography, elevated serum creatinine kinase levels, and muscle biopsy histopathology may also allude to the diagnosis. ${ }^{2}$ Preconceptually, our patient had persistently elevated creatinine kinase of 699 and 1,113 units/L.

Desmin-related myopathy has been characterized by an abnormal accumulation of desmin within cardiac and skeletal muscle. ${ }^{2}$ Therefore, we would not expect it to significantly affect the uterine smooth muscle. Antenatally, our patient had no issues surrounding cervical shortening, preterm contractions, or preterm labor. Moreover, her intrapartum course was unremarkable-tocometer monitoring and labor partogram was not suggestive of uterine dysfunction. Although there was uterine atony following delivery, it responded to conventional therapy, was not difficult to control, and did not recur.

According to one review, less than 100 patients with desmin myopathy have been identified to date. ${ }^{2}$ Unfortunately, no specific treatment currently exists for DRCM, and individuals are managed on a symptomatic basis. Pathologic cardiac arrhythmias or advanced heart block represents an indication for pacemaker/defibrillator implantation. ${ }^{1,2}$ From a clinical perspective, DRCM mimics a restrictive cardiomyopathy process with preserved systolic function. Symptomatic restrictive cardiomyopathy generally carries a poor prognosis, with a 10 -year survival rate of $37 \%{ }^{10,11}$ Pregnancy is typically not advised in this population. ${ }^{10-12}$

There is a paucity of information on the optimal management of reproductive-aged women with desminopathy, and little evidence exists in the literature to guide physicians with respect to counseling these women on pregnancy-related outcomes. This report describes the first case of pregnancy in a patient with symptomatic DRCM. Although complicated by repeated inpatient admissions and postpartum hemorrhage, the pregnancy continued until term without apparent permanent deterioration in cardiac function, suggesting that this condition does not appear to represent an absolute contraindication to pregnancy. 


\section{Source of Work}

This source of this study is a case report.

\section{Disclosures}

The authors did not report any potential conflicts of interest.

\section{Funding}

Not applicable.

\section{Paper part of a presentation}

No.

\section{Acknowledgment}

None.

\section{References}

1 Piñol-Ripoll G, Shatunov A, Cabello A, et al. Severe infantile-onset cardiomyopathy associated with a homozygous deletion in desmin. Neuromuscul Disord 2009;19(6):418-422

2 Goldfarb LG, Vicart P, Goebel HH, Dalakas MC. Desmin myopathy. Brain 2004;127(Pt 4):723-734

3 Goldfarb LG, Dalakas MC. Tragedy in a heartbeat: malfunctioning desmin causes skeletal and cardiac muscle disease. J Clin Invest 2009;119(7):1806-1813
4 Goldfarb LG, Olivé M, Vicart P, Goebel HH. Intermediate filament diseases: desminopathy. Adv Exp Med Biol 2008; 642:131-164

5 Taylor MR, Slavov D, Ku L, et al; Familial Cardiomyopathy Registry; BEST (Beta-Blocker Evaluation of Survival Trial) DNA Bank. Prevalence of desmin mutations in dilated cardiomyopathy. Circulation 2007;115(10):1244-1251

6 Sung RK, Ursell PC, Rame JE, et al. QTc prolongation and family history of sudden death in a patient with desmin cardiomyopathy. Pacing Clin Electrophysiol 2011;34(12):e105-e108

7 Franklin WJ, Benton MK, Parekh DR. Cardiac disease in pregnancy. Tex Heart Inst J 2011;38(2):151-153

8 Yancy CW, Jessup M, Bozkurt B, et al. 2013 ACCF/AHA guideline for the management of heart failure: executive summary: a report of the American College of Cardiology Foundation/American Heart Association Task Force on practice guidelines. Circulation 2013; 128(16):1810-1852

9 Zhang J, Kumar A, Stalker HJ, et al. Clinical and molecular studies of a large family with desmin-associated restrictive cardiomyopathy. Clin Genet 2001;59(4):248-256

10 Ammash NM, Seward JB, Bailey KR, Edwards WD, Tajik AJ. Clinical profile and outcome of idiopathic restrictive cardiomyopathy. Circulation 2000;101(21):2490-2496

11 Krul SP, van der Smagt JJ, van den Berg MP, Sollie KM, Pieper PG, van Spaendonck-Zwarts KY. Systematic review of pregnancy in women with inherited cardiomyopathies. Eur J Heart Fail 2011; 13(6):584-594

12 Herrey AS. Pregnancy in inherited and acquired cardiomyopathies. Best Pract Res Clin Obstet Gynaecol 2014;28(4):563-577 